\title{
Metaplasticity in the human swallowing system: clinical implications for dysphagia rehabilitation
}

\author{
Ivy Cheng ${ }^{1}$ (i) $\cdot$ Shaheen Hamdy ${ }^{1}$ (i)
}

Received: 18 March 2021 / Accepted: 5 October 2021 / Published online: 16 October 2021

(c) The Author(s) 2021

\begin{abstract}
Dysphagia is a common and devastating complication following brain damage. Over the last 2 decades, dysphagia treatments have shifted from compensatory to rehabilitative strategies that facilitate neuroplasticity, which is the reorganization of neural networks that is essential for functional recovery. Moreover, there is growing interest in the application of cortical and peripheral neurostimulation to promote such neuroplasticity. Despite some preliminary positive findings, the variability in responsiveness toward these treatments remains substantial. The purpose of this review is to summarize findings on the effects of neurostimulation in promoting neuroplasticity for dysphagia rehabilitation and highlight the need to develop more effective treatment strategies. We then discuss the role of metaplasticity, a homeostatic mechanism of the brain to regulate plasticity changes, in helping to drive neurorehabilitation. Finally, a hypothesis on how metaplasticity could be applied in dysphagia rehabilitation to enhance treatment outcomes is proposed.
\end{abstract}

Keywords Dysphagia $\cdot$ Metaplasticity $\cdot$ Neurostimulation $\cdot$ Neuroplasticity $\cdot$ Rehabilitation $\cdot$ Swallowing

\section{Introduction}

Recovery from brain damage such as stroke is thought to depend, in part, on neuroplasticity, which can be defined as the ability of the nervous system to re-organize its function, structure, and connections in response to intrinsic and extrinsic stimuli [1]. It can occur during development, learning, and in response to brain disruptions or to therapy [1]. Research on rehabilitation of swallowing function after brain injury has started to focus on strategies that promote such plasticity. Neurostimulation techniques, including non-invasive brain stimulation (NIBS) and stimulatory approaches that target the afferent neural pathways of swallowing, have been studied vigorously in recent years and the findings are encouraging [2]. However, there is substantial variability in the responsiveness toward these treatments. Genetic predisposition, brain configuration, and level of neural activation prior to neurostimulation are some of factors that may

Ivy Cheng

ivy.cheng@manchester.ac.uk

1 Centre for Gastrointestinal Sciences, Division of Diabetes, Endocrinology and Gastroenterology, School of Medical Sciences, Faculty of Biology, Medicine and Health, University of Manchester, Manchester, UK account for such variability [3]. Among these factors, the neural activation of the motor cortex may be programmed or preconditioned with additional neurostimulation through homeostatic metaplasticity, which refers to the regulation of changes in plasticity [4]. This offers unique opportunities in which the brain could be externally manipulated to achieve less variable treatment outcomes. The present review introduces the mechanisms of neuroplasticity and metaplasticity, summarizes findings on the use of neurostimulation to induce these processes, and discusses the potential application of metaplasticity to improve dysphagia treatment outcomes.

\section{Neuroplasticity: a key to functional recovery following brain injury}

Synaptic plasticity is a form of neuroplasticity. Donald Olding Hebb first proposed that repeated firing of one neuron would result in firing of another functionally connected neuron, leading to an increase in synaptic efficiency [5]. Such synaptic plasticity (Hebbian plasticity) is crucial for establishing new or reinforcing neural connections for functional recovery following stroke [6]. It can occur through longlasting activity-dependent increases (long-term potentiation; 
LTP) or decreases (long-term depression; LTD) in synaptic strength [1].

Synaptic (Hebbian) plasticity is regulated by the timely interaction between presynaptic and postsynaptic activity. The direction of plasticity (LTP or LTD) depends on the activation of N-methyl-D-aspartate receptors (NMDARs) and the influx of calcium $\left(\mathrm{Ca}^{2+}\right)$ ions to the postsynaptic neuron [7]. NMDAR activation is maximal when inducing LTP. Following depolarization of presynaptic neuron, glutamate-containing synaptic vesicles bind with the presynaptic membrane and glutamate is released. Glutamate molecules then bind to NMDARs on the postsynaptic membrane, resulting in depolarization of postsynaptic membrane. Sufficient postsynaptic membrane depolarization would result in a release of magnesium $\left(\mathrm{Mg}^{2+}\right)$ ions that block NMDRs and allow influx of $\mathrm{Ca}^{2+}$ ions to the postsynaptic cell. This $\mathrm{Ca}^{2+}$ ion influx activates the $\mathrm{Ca}^{2+} /$ calmodulin-dependent kinase II (CaMKII) pathway and a cascade of intercellular responses that alter synaptic efficiency [8]. Conversely, a modest activation of NMDARs and hence a smaller influx of $\mathrm{Ca}^{2+}$ ion to the postsynaptic cell is more favorable for the induction of LTD [8].

A number of NIBS techniques, including repetitive transcranial magnetic stimulation (rTMS) and transcranial direct current stimulation (tDCS), have been used to induce longlasting plasticity changes in the human hand $[9,10]$ and pharyngeal motor cortex [11, 12]. Such synaptic plasticity changes may result in behavioral or functional changes critical for rehabilitation. Transcranial magnetic stimulation (TMS) is a technique that induces electrical current within the brain through electromagnetic induction. Such electrical currents induced by single pulses of TMS can transiently depolarize the postsynaptic membrane, resulting in action potentials and single-pulse TMS is frequently used as a measure of corticobulbar or corticospinal connectivity [13]. When TMS is applied repetitively (repetitive TMS; rTMS), it can increase or decrease cortical excitability beyond the duration of the stimulation period. For tDCS, direct currents delivered through electrodes on the scalp can penetrate the skull to the brain and modify transmembrane potentials, thereby also changing cortical excitability [10]. In general, high-frequency $(>1 \mathrm{~Hz})$ rTMS and anodal tDCS induce LTP-like plasticity whereas low-frequency $(\leq 1 \mathrm{~Hz})$ rTMS and cathodal tDCS induce LTD-like plasticity in the human motor cortex.

Although plasticity plays an important role in brain development and in functional recovery following brain injury, there is a need for checks and balances in the nervous system because synaptic strengthening can be boundless and may result in excitotoxicity by over-excitation of NMDARs and high concentration of neurotoxins that causes cell death [14, 15]. On the other hand, saturation of LTD will lead to synaptic inactivity that compromises the ability of the neural networks to adapt to changes $[14,15]$. Therefore, mechanisms to balance and modulate these plasticity changes are necessary.

\section{Homeostatic metaplasticity: the regulator of plasticity}

Homeostatic metaplasticity is one such mechanism that regulates plasticity changes and maintains equilibrium at the level of neural activity [4]. This homeostatic mechanism stabilizes the activities of neurons and hence prevents saturation of LTP or LTD within the neural system [15]. Through metaplasticity, the preceding state of synaptic or neural activity can influence the characteristics of the subsequent synaptic changes $[15,16]$. Metaplasticity can be explained by the Bienenstock-Cooper-Munro (BCM) computational model [17] (Fig. 1). This model suggested that activity-dependent synaptic activity has a dynamic threshold, which could be modified by preceding postsynaptic activity. The LTD/LTP crossover threshold, termed "modification threshold," is reduced when there has been preceding low level postsynaptic activity and is increased when there has been preceding high level postsynaptic activity [15-17]. In a study with rat brain slices, Huang et al. [18] demonstrated this phenomenon in the hippocampus. When the synapse was first given either weak tetanic stimulation or single strong shocks, followed by an LTP-inducing stimulation, the LTP induced by the subsequent stimulation were reduced or inhibited. This likely resulted from the activation of NMDARs in response to the first stimuli. The authors postulated that this dynamic influence on the threshold of LTP can prevent a positive feedback loop and hence reduce over-excitation of synapses.

The underlying cellular mechanism for such dynamic influence remains poorly understood, but it is generally accepted that this form of metaplasticity is regulated by the ratio of NMDAR subunits (NR2A/NR2B ratio). NR2A is associated with fast kinetics of excitatory postsynaptic currents whereas NR2B subunit is associated with slow kinetics and strong cellular $\mathrm{Ca}^{2+}$ ion entry and LTP [19]. With elevated NR2A/NR2B ratio, the modification threshold is high and stronger stimulation is required to induce LTP whereas with a reduced ratio, weaker stimulation is adequate to induce LTP [7].

\section{Evidence of metaplasticity from animal and human studies}

The BCM model provides the basis for the application of NIBS techniques to induce metaplasticity in human motor cortex. It is hypothesized that preconditioned stimulation can shape the effects of the subsequent stimulation [15]. Iyer et al. [20] first explored metaplasticity properties in the human motor cortex in 25 healthy volunteers. They 
Fig. 1 Graphic representation of the Bienenstock-Cooper-Munro (BCM) model. The BCM model states that prior high level of postsynaptic activity lowers the threshold for LTD $\left(\Theta_{\text {LTD }}\right)$ and raises that for LTP $\left(\Theta_{\mathrm{LTP}}\right)$. The converse effect occurs with low previous level of activity

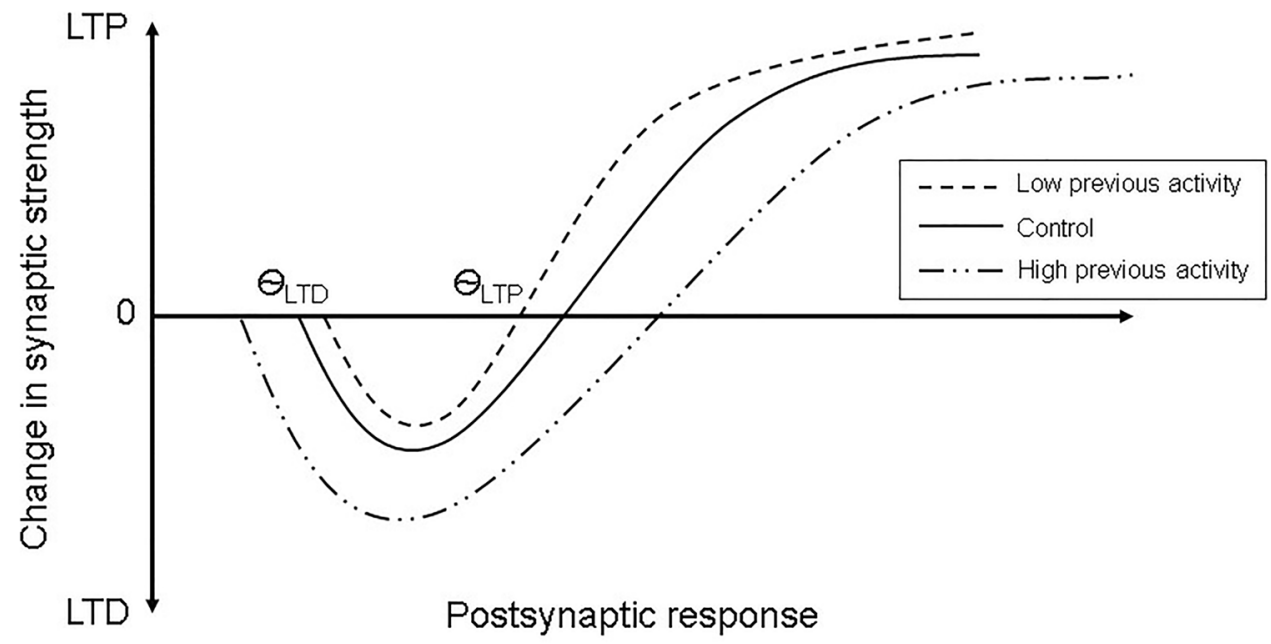

compared the preconditioning effects of constant frequency $(6 \mathrm{~Hz})$, frequency-modulated (ranging from 4 to $8 \mathrm{~Hz}$ ), and sham 6-Hz (high-frequency; excitatory) rTMS on plasticity changes induced by $1-\mathrm{Hz}$ (low-frequency; inhibitory) rTMS on the hand motor cortex. Their results showed that a short bout of 6-Hz rTMS, regardless of constancy of frequency, provoked stronger and longer suppression of cortical excitability induced by $1-\mathrm{Hz}$ rTMS compared to no preconditioning. This $1 \mathrm{~Hz}$-preconditioned $6-\mathrm{Hz}$ protocol has been tested later in a small group of stroke patients for its safety [21]. No major adverse effects were reported. However, the efficacy of such preconditioned protocol for stroke rehabilitation remained limited and therefore required further investigations.

Apart from using rTMS, different combinations of NIBS techniques, including tDCS, theta burst stimulation (TBS), and quadri-pulse stimulation (QPS), have been used to study metaplasticity of the hand motor cortex in healthy adults [22]. In all combinations, there was evidence that the state of cortical excitability preceding NIBS can be controlled within a reasonable physiological range using preconditioned stimulation.

\section{Promoting neuroplasticity in swallowing motor cortex through neurostimulation}

In early animal studies, Sumi [23] showed that chewing and swallowing responses can be elicited by applying electrical pulses to areas that are anterolateral in the frontal lobe or rostrolateral to the postcentral area of the cortex of anaesthetized rabbits. The study found that both swallowing and chewing are bilaterally innervated within the central nervous system. Interestingly, additional stimulation from the superior laryngeal nerves facilitated cortically induced swallowing, suggesting the bi-directional synergetic effects between sensory inputs and motor control of swallowing. A later study with awaked primates demonstrated that swallowing and orofacial muscles are controlled and mediated by distinct regions of the cerebral cortex using intracortical microstimulation [24]. These regions included the lateral region of face primary motor cortex (face-MI), the lateral face primary somatosensory cortex (face-SI), the area lateral and anterior to face-MI corresponding to the cortical masticatory area (CMA), and a deep cortical area corresponding to the white matter underlying the CMA and the frontal operculum [24]. These findings along with a number of other animal-based physiologic studies of swallowing [25] showed the swallowing motor system, unlike the limb motor system, is innervated by both hemispheres and is controlled by specific areas of the cerebral cortex.

Following the development of TMS, researchers began to explore the cortical representation of human swallowing musculature in awake human subjects non-invasively [26, 27]. Aziz et al. [26] showed that both early and late electromyographic responses could be elicited in the human esophagus following single-pulse TMS and these responses were probably mediated by two different neurological pathways because of the distinct neurophysiological characteristics. The early responses might be mediated by a paucisynaptic pyramidal pathway from frontoparietal cortex to brainstem reticular formation whereas the late responses might be mediated by polysynaptic extrapyramidal pathways that were involved in swallowing control [26]. A further study by Hamdy et al. [27] showed that human swallowing musculature, including pharyngeal, esophageal, and mylohyoid muscles, are discretely and somatotopically represented in the motor and premotor cortex. Mylohyoid muscles have been reported to be represented in the lateral precentral and inferior frontal gyri, whereas pharyngeal and esophageal muscles are represented in the anterolateral precentral and middle 
frontal gyri and in the anterolateral precentral and superior frontal gyri respectively [27]. These representations are bilateral but asymmetrical in the two hemispheres, independent of handedness. Moreover, studies using PET and fMRI showed that swallowing recruits multiple cerebral regions, predominantly in sensorimotor areas of the brain $[28,29]$. Most recently, Hashimoto et al. demonstrated for the first time the brain oscillation changes between voluntary and involuntary swallowing with electrocorticogram (ECoG) [30]. They found that the swallowing driving force switched from the cortex to brain stem at the transition from oral to pharyngeal phase of swallowing, providing evidence of the dynamics within the neural network of swallowing.

The evidence of plasticity in human swallowing motor cortex can be seen in patients who had dysphagia after stroke. Majority of the patients recover from dysphagia within weeks of stroke episode. This remarkable ability to restore swallowing function is believed to be driven by plasticity. In the study by Hamdy et al. [27], the changes in cortical pharyngeal representations of two stroke patients, one with dysphagia who fully recovered after 3 months and one without dysphagia, were examined at presentation and 3 months after stroke. They observed that at stroke presentation, the dysphagic patient had a smaller area of pharyngeal representation in the unaffected hemisphere than the non-dysphagic patient. This suggested that dysphagia may be resulted from damage to the hemisphere with larger representation (dominant hemisphere) such that the inputs from the unaffected, non-dominant hemisphere remain inadequate for functional swallowing. Moreover, the recovery of swallowing is related to an increase in the size of pharyngeal representation [27]. This study provided new evidence that plastic changes within the neural network for swallowing occur in response to a damage and play a critical role in functional recovery. Such observations were later corroborated by a further longitudinal TMS study [31]. The cortical representation of the pharynx in 28 unilateral hemispheric stroke patients was investigated at 1 week, and at 1 and 3 months after stroke. Among patients who had dysphagia initially, $75 \%$ recovered within first month after stroke. This recovery was associated with an increase in the pharyngeal representation of the unaffected hemisphere.

Taken together, these findings showed that swallowing musculature are asymmetrically represented in the two hemispheres and damage to the dominant hemisphere is likely to result in dysphagia. Importantly, recovery of swallowing function following unilateral stroke is driven by compensatory reorganization of the intact hemisphere. Facilitating such reorganization could therefore be a viable treatment goal for neurostimulation.

\section{Peripheral neurostimulation}

The findings on the course of recovery in stroke patients serve as a model for the development of dysphagia treatments that drive plasticity changes in dysphagic patients. This can be achieved via two forms of neurostimulation: peripheral or central neurostimulation. Peripheral neurostimulation targets the afferent pathways for swallowing whereas central neurostimulation targets the central brain control of swallowing. The swallowing neuroplastic effects of human peripheral neurostimulation were first explored by Hamdy et al. [32], who studied the changes in cortical excitability in 8 healthy adults following intraluminal pharyngeal electrical stimulation (PES). The results showed that sensory inputs from electrical stimulation of pharyngeal muscles could drive an increase in cortical excitability that lasted longer than the stimulation duration. It is likely that the sensory fibers in cranial nerves IX (glossopharyngeal) and X (vagus) were activated by PES [33]. In a further study, Fraser et al. [34] identified that there was frequency specificity to the stimulation, which may be related to the conduction time for sensory inputs from the pharynx to reach the sensorimotor cortex and the optimal time window for persistent depolarization of neuronal membrane to occur. This study also verified cortical excitation with fMRI results showing increased cortical activation in the swallowing network after PES. Moreover, it showed that in acute stroke patients with dysphagia, PES could increase pharyngeal cortical excitability of the intact hemisphere, which was associated with functional recovery of swallowing.

There are limited data on the effects of PES for patients with other neurological disease. A small randomized controlled trial found that PES could reduce penetration and aspiration in patients with multiple sclerosis [35]. Moreover, a recent multi-center open-label cohort study showed that PES improves swallowing in patients with neurogenic dysphagia associated with stroke, traumatic brain injury, mechanical ventilation, or tracheostomy [36].

Apart from electrical stimulation, sensory stimulation that involves the senses of smell [37], taste [38], and vision [39] has also been employed in an attempt to alter cortical excitability or activation of the swallowing motor cortex. Ebihara et al. [37] found that prolonged (30 days) nasal inhalation of black pepper oil could induce plasticity changes in the left insular cortex, as revealed by single-photon emission computed tomography, and improve the swallowing reflex in patients with post-stroke dysphagia. Mistry et al. [38] demonstrated that both sweet (glucose) and bitter (quinine) stimuli reduced cortical excitability of the pharyngeal motor cortex. In another study by Abdul Wahab et al. [40], the authors showed that cortical excitability could only be increased by a combination of, but not separately, olfactory and gustatory stimuli and such increase lasted for at least $90 \mathrm{~min}$. A further 
study by this group showed that such stimulation resulted in changes in swallowing biomechanics, including increase in duration and pressure of tongue-to-palate contact during swallowing [41]. Finally, food-related disgust pictures were found to reduce cortical excitability, possibly due to a triggering of avoidant-defensive mechanisms within the human swallowing system [39]. Taken together, olfactory, gustatory, and visual stimuli can either facilitate or suppress swallowing and such changes are mediated by the central nervous system.

Moreover, thermal and chemical stimulation could also alter cortical excitability of the swallowing motor cortex. Studies have shown that cold $\left(15^{\circ} \mathrm{C}\right)$ thermal stimulation on the tongue could increase excitability of the pharyngeal motor cortex, providing evidence of interaction between the tongue and pharyngeal cortical areas and between the afferent and efferent neural pathways [42, 43]. Moreover, a study by Elshukri et al. [44] found that carbonated boluses could increase cortical excitability that lasted for up to $60 \mathrm{~min}$ following the bolus inputs. Recently, a study using highresolution manometry found that cold, sour, and carbonated boluses could increase pharyngeal contraction during swallowing in dysphagic patients [45].

Taken together, these neurophysiological findings suggest that the sensory neural pathways of swallowing could be manipulated through peripheral neurostimulation to facilitate plasticity changes in the motor cortex, making neurostimulation methods such as these a potentially viable technique for dysphagia treatment.

\section{Central (cortical and cerebellar) neurostimulation}

Studies have shown that central neurostimulation using NIBS, including tDCS and rTMS, can induce plastic changes in human swallowing (pharyngeal) motor cortex of healthy individuals $[12,46]$. An early study by Jefferson et al. [47] demonstrated that anodal (excitatory) tDCS (1 mA for $20 \mathrm{~min}$ or $1.5 \mathrm{~mA}$ for $10 \mathrm{~min}$ ) could induce long-lasting increases in cortical excitability in healthy participants. Similarly, rTMS was found to be able to induce LTP- and LTD-like plasticity changes in the pharyngeal motor cortex. Gow et al. [46] found that the induction of plasticity is frequency-specific, with 5-Hz rTMS eliciting the most significant and longest-lasting increase in the human pharyngeal motor cortex (up to $1 \mathrm{~h}$ ) after stimulation. For lowfrequency rTMS, Mistry et al. [12] found that $1-\mathrm{Hz}$ rTMS presented at $120 \%$ pharyngeal resting motor threshold could reduce cortical excitability in pharyngeal motor cortex of the stimulated hemisphere, an effect that lasted for up to $45 \mathrm{~min}$. Moreover, stimulation of the dominant, but not the nondominant, pharyngeal hemisphere could temporarily disrupt swallowing behavior. Their findings showed that $1-\mathrm{Hz}$ rTMS can be used to induce a "virtual lesion" in healthy adults, which provides a model that mimics disruption of swallowing motor pathways after brain damage. The virtual lesion approach thus allows investigation of potential treatment strategies for patients with swallowing disorders. Several studies have shown that both peripheral and central neurostimulation could be applied to reverse this virtual lesion in the pharyngeal motor cortex [11,48-50], giving credence to their likely usefulness in patient populations.

Over the past decade, clinical studies have demonstrated positive effects of NIBS in patients with post-stroke dysphagia [2]. Low-frequency rTMS has been used to suppress cortical excitability of the unaffected hemisphere to reduce interhemispheric inhibition [51-54], while high-frequency rTMS applied to the affected hemisphere was used to increase cortical excitability and overcome interhemispheric imbalance $[52,55]$. Moreover, studies have shown that by increasing cortical excitability of the unaffected hemisphere, it is possible to improve swallowing function, presumably through facilitating reorganization of the compensatory neural network [50, 56, 57]. Some studies explored $10-\mathrm{Hz}$ (high-frequency) rTMS over both hemispheres and found that bilateral rTMS improved swallowing more significantly than unilateral rTMS or sham rTMS [58-60]. Unlike rTMS, tDCS protocols are less diverse. Almost all studies in the literature employed anodal (excitatory) tDCS, over either the affected hemisphere [61-63], unaffected hemisphere $[64,65]$, or both hemispheres [66, 67]. A recent systematic review suggested that cortical neurostimulation is beneficial in improving swallowing in stroke patients [2]. Of note, studies have shown positive effects in both excitatory and inhibitory stimulation of the unaffected hemisphere. These seemingly contradictory outcomes may reveal the impact of stroke severity on the response to brain stimulation. It is possible that patients with severe stroke may be more responsive to stimulation that promotes reorganization of compensatory neural network (excitatory stimulation on unaffected hemisphere). Contrarily, those with less severe stroke may be more responsive to stimulation that lowers interhemispheric inhibition and allows reorganization of the residual neural networks within the affected hemisphere (inhibitory stimulation on unaffected hemisphere). Moreover, studies have shown that chronic stroke patients may have maladaptive neuroplastic changes in the affected hemisphere that hinder recovery of swallowing function [56]. Therefore, inhibitory stimulation may be useful to suppress such changes. However, these explanations remain speculative without analysis of data stratified according to stroke severity and chronicity. Importantly, bihemispheric stimulation appeared to yield better outcomes compared to unihemispheric stimulation (Fig. 2). This agrees with animal data which showed that electrical stimulation on both hemispheres elicited more frequent swallowing than the sum of swallows elicited separately from stimulation on each hemisphere [23]. 
Bihemispheric stimulation may have synergetic effects and result in more significant plasticity changes and functional recovery.
Similar to peripheral neurostimulation, there are limited data on the effects of central neurostimulation on dysphagic patients with other neurological diseases. A pilot study showed that 5-Hz rTMS improved swallowing and cortical
Fig. 2 Simplified forest plot adapted from Cheng et al. [2] showing treatment effects of cortical neurostimulation based on stimulation hemisphere, including affected hemisphere, unaffected hemisphere, and both hemispheres, on swallowing functions in stroke patients. All three approaches showed beneficial effects when compared with control treatment (conventional dysphagia therapy or sham neurostimulation). Bihemispheric stimulation had the largest pooled effect size among all approaches

\section{Std. Mean Difference N, Random, $95 \% \mathrm{Cl}$}

Stucty or Subgroup

(I) Affected hemisphere

Cheng 2017

Du 2016 a

E Park 2013b

Khedr 2009

Kim 2011a

Pingue 2018

Shigematsu 2013

Yang 2012

Zhang 2019a

Subtotal $(95 \% \mathrm{Cl})$

(l) Unaffected hemisphere

Cabib 2020

Du 2016b

Kim 2011b

Kumar 2011

Lim 2014

Michou 2014

Park 2013

Suntrup-Krueger 2018

Tarameshlu 2019a

Tarameshlu 2019b

Unluer 2019

Zhang 2019b

Subtotal $(95 \% \mathrm{Cl})$
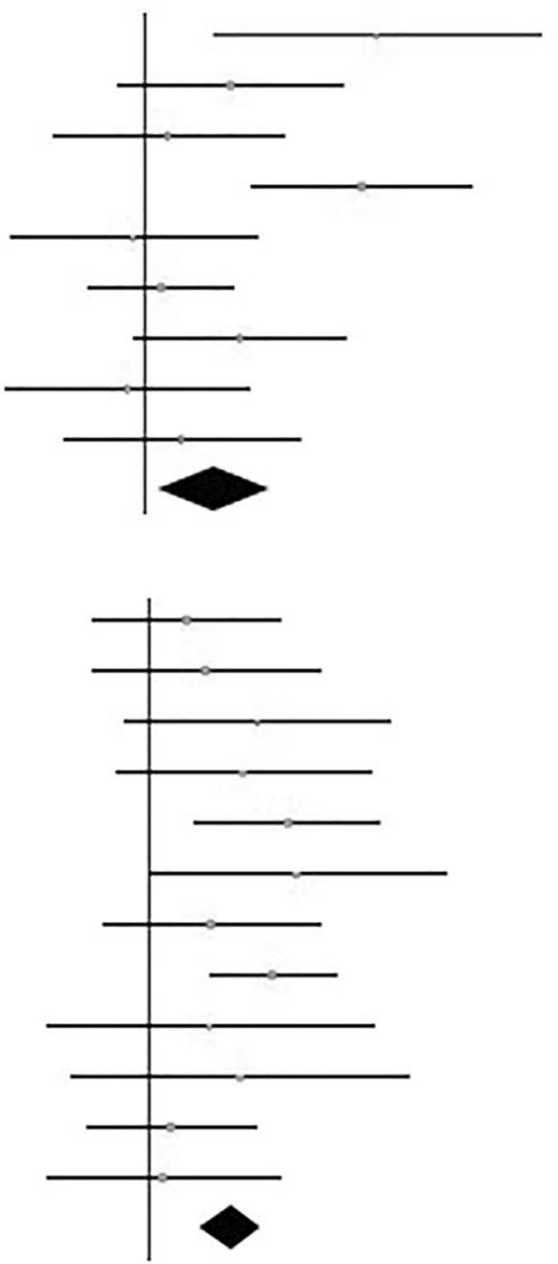

(l) Both hemispheres

Ann 2017

E Park 2013a

Khedr 2010a

Khedr 2010b

Wang 2020

Zhang $2019 \mathrm{C}$

Subtotal $(95 \% \mathrm{Cl})$

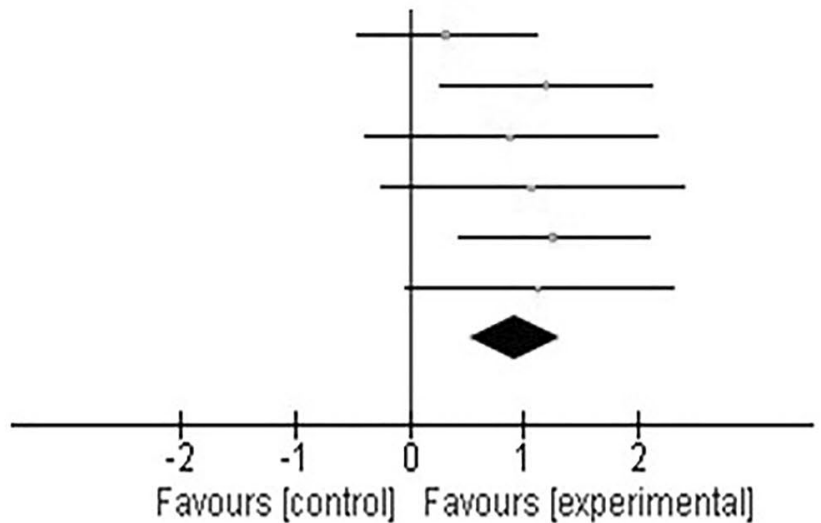


activation in elderly patients with dysphagia [68]. Moreover, a prospective controlled trial found that both tDCS and intermittent theta burst stimulation (iTBS), which is a form of patterned TMS and is considered excitatory, had beneficial effects in elderly patients with dysphagia [69]. In patients with Parkinson's disease, sequential application of $20-\mathrm{Hz}$ rTMS to both hemispheres for 10 days followed by 5 booster sessions every month for 3 months reduced dysphagia severity and improved pharyngeal transit time and hyoid elevation [70]. The rTMS protocol used in this study was different from other dysphagia studies in several ways. Firstly, the stimulation frequency was higher $(20 \mathrm{~Hz})$ than other highfrequency studies which usually used $5-\mathrm{Hz}$ or $10-\mathrm{Hz}$ rTMS. Secondly, there were booster sessions, which have not been explored in other trials. Finally, rTMS was applied over the hand motor cortex instead of swallowing motor cortex. The authors argued that the improvements may be due to spreading of excitation to the adjacent esophageal motor cortex, or enhancement of functional connectivity of swallowing network by stimulation of the primary motor cortex [70]. Apart from non-invasive brain stimulation, studies have also investigated the effects of deep brain stimulation (DBS) on swallowing function in patients with Parkinson's disease. However, the results were conflicting and there was not enough evidence to show the effectiveness of DBS in improving swallowing [71]. Finally, a small randomized controlled trial demonstrated that tDCS reduced dysphagia severity and increased cortical excitability in patients with multiple sclerosis [72].

Apart from cortical stimulation, some studies have also explored the effects of stimulation on the cerebellum, which has been shown involved in swallowing [73-75]. Physiological studies with healthy volunteers have shown that cerebellar rTMS could modulate excitability of the pharyngeal motor cortex [76] and 10-Hz rTMS appeared to be optimal for increasing such cortical excitability [77]. A further study found that $10-\mathrm{Hz}$ rTMS could reverse the neurological and behavioral disruptions caused by a "virtual lesion" of the pharyngeal motor cortex [78]. Similar to cortical stimulation, bilateral cerebellar stimulation showed stronger facilitatory effects compared to unilateral stimulation [79]. Interestingly, the effects of cerebellar neurostimulation appear to be site-specific. Studies have found that rTMS or tDCS applied over the midline of cerebellum result in suppressive effects on pharyngeal cortical excitability and swallowing behavior or skill learning [80, 81]. While cerebellar neurostimulation may appear to have therapeutic potential for dysphagia, most of the data come from healthy volunteer studies. A single-patient case-controlled study found that cerebellar rTMS improved swallowing safety and increased cortical excitability in a patient with right posterior fossa infarction [82]. Recently, a quasi-randomized controlled trial found that 5-Hz suprathreshold (110\% mylohyoid motor threshold)
rTMS applied over the cerebellum showed beneficial effects on swallowing function in patients with post-stroke dysphagia comparable to rTMS applied over affected or unaffected hemispheres [83]. Further studies are warranted to fully explore the clinical efficacy of cerebellar neurostimulation in dysphagic patients.

Despite these positive findings, substantial variability in the responses toward NIBS treatments has been reported [84]. This prevents a generalized application and translation into clinical practice. The possible factors for such variability include genetic predisposition, brain configuration, and brain state prior to stimulation [3]. Given the intrinsic metaplastic mechanisms of the brain that regulates its response to plasticity-inducing stimulation, it is reasonable to hypothesize that preconditioning of the motor cortex prior to neurostimulation could minimize the response variability and subsequently enhance treatment outcomes.

\section{Metaplasticity in human swallowing motor cortex: preliminary evidence}

Very few studies have explored metaplasticity mechanism in the human swallowing motor cortex. An early study by Michou et al. [85] found that "non-responders" to paired associative stimulation (PAS), which is a form of NIBS, can be switched to "responders" by delivering an additional dose of PAS within an hour. This result could not be explained by the BCM model of metaplasticity, in which double exposure of excitatory stimulation should have resulted in an overall neutral or inhibitory response. The authors suggested that the capacity or threshold for triggering a metaplasticity mechanism in the swallowing system has not been wellstudied and that the protocols employed may not be the most appropriate for inducing metaplasticity. Moreover, the interPAS interval may play a role in affecting the results. It is also possible that the non-responders had opposite response to excitatory stimulation (inhibition) such that the first stimulation had artificially preconditioned the motor cortex into an inhibited state and lowered its threshold for LTP. When the motor cortex received the second dose of stimulation, LTP (expected response) was induced due to metaplasticity.

Recently, Cheng et al. [86] explored the induction of metaplasticity in the human swallowing motor cortex. They compared different preconditioned rTMS protocols (excitatory: $5-\mathrm{Hz}$ rTMS preconditioned with $1-\mathrm{Hz}$ rTMS; and inhibitory: $1-\mathrm{Hz}$ rTMS preconditioned with $5-\mathrm{Hz}$ rTMS) with varying inter-stimulation interval in 28 healthy volunteers. Their study showed that the pharyngeal motor cortex exhibited bi-directional metaplasticity properties in which inhibitory and excitatory effects of rTMS on cortical excitability and swallowing behavior could be enhanced by preconditioned rTMS. Moreover, there appeared to be a critical 
period within which metaplasticity could be induced. Specifically, the optimal inter-stimulation interval was $30 \mathrm{~min}$ for the 5-Hz rTMS preconditioned with 1-Hz rTMS protocol and $90 \mathrm{~min}$ for the $1-\mathrm{Hz}$ rTMS preconditioned with $5-\mathrm{Hz}$ rTMS protocol. This suggests that metaplastic changes could only take place when the after-effects of the preconditioning rTMS had built up to an adequate level. This study is an important milestone for dysphagia rehabilitation because it provides evidence that metaplasticity in the human swallowing system is functionally relevant and can be harnessed therapeutically. These findings encourage further investigations as to whether such homeostatic metaplasticity exists in patients with neurogenic dysphagia and how treatment outcomes can be improved through preconditioning of the swallowing motor cortex.

\section{Future research direction: cross-modal preconditioning}

Apart from cortical stimulation, the motor cortex may potentially be preconditioned with other forms of plasticity-inducing stimulation, for example, peripheral (electrical, sensory, chemical, or thermal) neurostimulation. Moreover, it is not known whether preconditioning the motor cortex before traditional dysphagia therapy (that might involve behavioral exercises) would result in better treatment outcomes. Although little is known in the field of dysphagia rehabilitation, we could hypothesize the clinical usefulness of cross-modal preconditioned treatments based on the evidence from limb motor function rehabilitation trials. For example, studies have shown that plasticity induced by motor training can be enhanced by eliciting homeostatic metaplasticity [19]. Prior to motor training, neural activity could be suppressed by cortical stimulation to lower the threshold for LTP-like plasticity. Indeed, Ziemann et al. [87] found that better outcomes on a thumb abduction movement training could be achieved when participants have received LTD-like plasticity-inducing brain stimulation prior to the training than without preconditioning. Recently, a study with Parkinson's disease patients also demonstrated that the outcomes of motor learning were more significant and sustainable after preconditioning with rTMS compared to without preconditioning [88].

Given these encouraging results from limb motor function studies, it seems reasonable to hypothesize that similar principles may be applicable to swallowing exercise training. Behavioral rehabilitative exercises, including strength training, skill-based training, and combinatory approaches, have shown positive physiological effects [89, 90]. Of relevance, Malandraki et al. [91] reported that an 8-week lingual resistance exercise regime could increase cortical activation during swallowing and lingual pressure in a chronic stroke patient. At the initial phase of training, the patient displayed perilesional activity in the affected hemisphere. However, after 8 weeks of training, there was an increase in activation in primary motor cortex, primary sensory cortex, premotor area, and insula in both hemispheres and such changes were associated with increased lingual pressure. This finding suggested behavioral exercises could induce reorganization of the neural network.

Linking the above findings to the future application of metaplasticity to swallowing rehabilitation, some studies have suggested that NIBS (tDCS and rTMS) combined with dysphagia therapy, which involves behavioral exercises, is superior to therapy alone in improving swallowing in stroke patients [53, 54, 92, 93]. While we cannot be certain about the underlying mechanism that drove this superiority, and whether cortical neurostimulation acted as prelude to inducing metaplasticity and in preparing the swallowing system for other therapeutic inputs, these findings demonstrated that when cortical neurostimulation is combined with behavioral therapy, the outcomes could be synergised. This sheds lights on the therapeutic potential of cross-modal treatments. Future studies may investigate the underlying mechanisms by manipulating other parameters of the protocol, for example, the interval between the two forms of treatments, and whether excitatory and inhibitory stimulation preceding behavioral therapy would result in opposite outcomes. Moreover, given the neurophysiological findings of peripheral (sensory, electrical, chemical, and thermal) neurostimulation, it would be interesting to investigate whether these methods could also be used as preconditioning inputs before cortical neurostimulation, or vice versa, to induce metaplasticity and enhance overall treatment outcomes.

\section{Conclusions}

Rehabilitation of neurogenic dysphagia is thought to be mainly driven by neuroplasticity, which involves modulation of synaptic strength. Studies have shown that both peripheral and central (cortical and cerebellar) neurostimulation treatments can promote such neuroplasticity in patients with neurogenic dysphagia. Peripheral neurostimulation is mainly used to increase sensory input to the central nervous system whereas central brain stimulation is used to directly induce plasticity changes in the cortex or cerebellum. However, the response to these techniques is highly variable, possibly due to genetic variability across individuals or variations in levels of brain activation prior to stimulation. Therefore, it is important to develop robust strategies to improve treatment outcomes. Recently, the concept of metaplasticity, which is the higher order brain function that regulates responses to plasticity changes, has been explored in the human swallowing motor system. There is preliminary evidence that 
the outcomes of brain stimulation can be enhanced through metaplasticity induced by preconditioning of the cortex. However, it is not yet known whether metaplasticity can be induced in patients with neurogenic dysphagia with similar preconditioned protocols. Nonetheless, the concept of metaplasticity allows further exploration on the effects of preconditioning, potentially through combinations of different modalities (peripheral or central), on the human swallowing motor system and its therapeutic values in promoting recovery of neurogenic dysphagia.

\section{Acknowledgements Not applicable}

Author contribution All authors contributed to the idea of the review article. IC performed literature search and drafted the manuscript. SH critically revised the work. All authors read and approved the final manuscript.

Data availability Not applicable.

Code availability Not applicable.

\section{Declarations}

Ethical standards statements No human or animal subjects were involved in this review article.

Informed consent None.

Conflict of interest The authors declare no competing interests.

Open Access This article is licensed under a Creative Commons Attribution 4.0 International License, which permits use, sharing, adaptation, distribution and reproduction in any medium or format, as long as you give appropriate credit to the original author(s) and the source, provide a link to the Creative Commons licence, and indicate if changes were made. The images or other third party material in this article are included in the article's Creative Commons licence, unless indicated otherwise in a credit line to the material. If material is not included in the article's Creative Commons licence and your intended use is not permitted by statutory regulation or exceeds the permitted use, you will need to obtain permission directly from the copyright holder. To view a copy of this licence, visit http://creativecommons.org/licenses/by/4.0/.

\section{References}

1. Ward NS, Cohen LG (2004) Mechanisms underlying recovery of motor function after stroke. Arch Neurol 61(12):1844-1848

2. Cheng I, Sasegbon A, Hamdy S (2020) Effects of neurostimulation on poststroke dysphagia: a synthesis of current evidence from randomized controlled trials. Neuromodulation. https://doi.org/10. $1111 /$ ner. 13327

3. Ridding MC, Ziemann U (2010) Determinants of the induction of cortical plasticity by non-invasive brain stimulation in healthy subjects. J Physiol 588(Pt 13):2291-2304

4. Abraham WC, Bear MF (1996) Metaplasticity: the plasticity of synaptic plasticity. Trends Neurosci 19(4):126-130
5. Hebb DO (1949) The organization of behavior. A neuropsychological theory. Wiley, New York

6. Takeuchi N, Izumi S (2015) Combinations of stroke neurorehabilitation to facilitate motor recovery: perspectives on Hebbian plasticity and homeostatic metaplasticity. Front Hum Neurosci 9:349

7. Yashiro K, Philpot BD (2008) Regulation of NMDA receptor subunit expression and its implications for LTD, LTP, and metaplasticity. Neuropharmacology 55(7):1081-1094

8. Luscher C, Malenka RC (2012) NMDA receptor-dependent longterm potentiation and long-term depression (LTP/LTD). Cold Spring Harb Perspect Biol 4(6):a005710

9. Fitzgerald PB, Fountain S, Daskalakis ZJ (2006) A comprehensive review of the effects of rTMS on motor cortical excitability and inhibition. Clin Neurophysiol 117(12):2584-2596

10. Stagg CJ, Nitsche MA (2011) Physiological basis of transcranial direct current stimulation. Neuroscientist 17(1):37-53

11. Jefferson $S$ et al (2009) Reversal of a virtual lesion in human pharyngeal motor cortex by high frequency contralesional brain stimulation. Gastroenterology 137(3):841-9 849 e1

12. Mistry S et al (2007) Unilateral suppression of pharyngeal motor cortex to repetitive transcranial magnetic stimulation reveals functional asymmetry in the hemispheric projections to human swallowing. J Physiol 585(Pt 2):525-538

13. Pascual-Leone A et al (1998) Study and modulation of human cortical excitability with transcranial magnetic stimulation. J Clin Neurophysiol 15(4):333-343

14. Yger P, Gilson M (2015) Models of metaplasticity: a review of concepts. Front Comput Neurosci 9:138

15. Abraham WC (2008) Metaplasticity: tuning synapses and networks for plasticity. Nat Rev Neurosci 9(5):387

16. Muller-Dahlhaus F, Ziemann U (2015) Metaplasticity in human cortex. Neuroscientist 21(2):185-202

17. Bienenstock EL, Cooper LN, Munro PW (1982) Theory for the development of neuron selectivity: orientation specificity and binocular interaction in visual cortex. J Neurosci 2(1):32-48

18. Huang YY et al (1992) The influence of prior synaptic activity on the induction of long-term potentiation. Science 255(5045):730-733

19. Ziemann U, Siebner HR (2008) Modifying motor learning through gating and homeostatic metaplasticity. Brain Stimul 1(1):60-66

20. Iyer MB, Schleper N, Wassermann EM (2003) Priming stimulation enhances the depressant effect of low-frequency repetitive transcranial magnetic stimulation. J Neurosci 23(34):10867-10872

21. Carey JR et al (2008) Safety of 6-Hz primed low-frequency rTMS in stroke. Neurorehabil Neural Repair 22(2):185-192

22 Hassanzahraee M, Zoghi M, Jaberzadeh S (2018) How different priming stimulations affect the corticospinal excitability induced by noninvasive brain stimulation techniques: a systematic review and meta-analysis. Rev Neurosci 29(8):883-899

23. Sumi T (1969) Some properties of cortically-evoked swallowing and chewing in rabbits. Brain Res 15(1):107-120

24. Martin RE et al (1999) Features of cortically evoked swallowing in the awake primate (Macaca fascicularis). J Neurophysiol 82(3):1529-1541

25. Miller AJ (1986) Neurophysiological basis of swallowing. Dysphagia 1(2):91

26. Aziz Q et al (1995) Topographic mapping of cortical potentials evoked by distension of the human proximal and distal oesophagus. Electroencephalogr Clin Neurophysiol 96(3):219-228

27. Hamdy $\mathrm{S}$ et al (1996) The cortical topography of human swallowing musculature in health and disease. Nat Med 2(11):1217-1224

28. Hamdy $\mathrm{S}$ et al (1999) Cortical activation during human volitional swallowing: an event-related fMRI study. Am J Physiol 277(1 Pt 1):G219-G225 
29. Hamdy $\mathrm{S}$ et al (1999) Identification of the cerebral loci processing human swallowing with H-2 O-15 PET activation. J Neurophysiol 81(4):1917-1926

30. Hashimoto $\mathrm{H}$ et al (2020) Motor and sensory cortical processing of neural oscillatory activities revealed by human swallowing using intracranial electrodes. bioRxiv

31. Hamdy S et al (1998) Recovery of swallowing after dysphagic stroke relates to functional reorganization in the intact motor cortex. Gastroenterology 115(5):1104-1112

32. Hamdy $\mathrm{S}$ et al (1998) Long-term reorganization of human motor cortex driven by short-term sensory stimulation. Nat Neurosci 1(1):64-68

33. Gow D et al (2004) Characterising the central mechanisms of sensory modulation in human swallowing motor cortex. Clin Neurophysiol 115(10):2382-2390

34. Fraser $\mathrm{C}$ et al (2002) Driving plasticity in human adult motor cortex is associated with improved motor function after brain injury. Neuron 34(5):831-840

35. Restivo DA et al (2013) Pharyngeal electrical stimulation for dysphagia associated with multiple sclerosis: a pilot study. Brain Stimul 6(3):418-423

36. Bath PM et al (2020) Pharyngeal electrical stimulation for neurogenic dysphagia following stroke, traumatic brain injury or other causes: main results from the PHADER cohort study. EClinicalMedicine 28:100608

37. Ebihara T et al (2006) A randomized trial of olfactory stimulation using black pepper oil in older people with swallowing dysfunction. J Am Geriatr Soc 54(9):1401-1406

38. Mistry S et al (2006) Modulation of human cortical swallowing motor pathways after pleasant and aversive taste stimuli. Am J Physiol Gastrointest Liver Physiol 291(4):G666-G671

39. Vicario CM et al (2017) Pictures of disgusting foods and disgusted facial expressions suppress the tongue motor cortex. Soc Cogn Affect Neurosci 12(2):352-362

40 Abdul Wahab N, Jones RD, Huckabee ML (2010) Effects of olfactory and gustatory stimuli on neural excitability for swallowing. Physiol Behav 101(5):568-75

41. Wahab NA, Jones RD, Huckabee ML (2011) Effects of olfactory and gustatory stimuli on the biomechanics of swallowing. Physiol Behav 102(5):485-490

42 Magara J et al (2020) Lasting modulation of human cortical swallowing motor pathways following thermal tongue stimulation. Neurogastroenterol Motil 33:e13938

43. Magara $\mathrm{J}$ et al (2018) Cold thermal oral stimulation produces immediate excitability in human pharyngeal motor cortex. Neurogastroenterol Motil 30(10):e13384

44. Elshukri $\mathrm{O}$ et al (2016) Brain and behavioral effects of swallowing carbonated water on the human pharyngeal motor system. J Appl Physiol (1985) 120(4):408-15

45. Regan J (2020) Impact of sensory stimulation on pharyngoesophageal swallowing biomechanics in adults with dysphagia: a high-resolution manometry study. Dysphagia 35(5):825-833

46. Gow D et al (2004) Induction of long-term plasticity in human swallowing motor cortex following repetitive cortical stimulation. Clin Neurophysiol 115(5):1044-1051

47. Jefferson $\mathrm{S}$ et al (2009) Characterizing the application of transcranial direct current stimulation in human pharyngeal motor cortex. Am J Physiol Gastrointest Liver Physiol 297(6):G1035-G1040

48. Vasant DH et al (2014) Transcranial direct current stimulation reverses neurophysiological and behavioural effects of focal inhibition of human pharyngeal motor cortex on swallowing. J Physiol 592(4):695-709

49. Jayasekeran V et al (2010) Adjunctive functional pharyngeal electrical stimulation reverses swallowing disability after brain lesions. Gastroenterology 138(5):1737-1746
50. Michou E et al (2012) Targeting unlesioned pharyngeal motor cortex improves swallowing in healthy individuals and after dysphagic stroke. Gastroenterology 142(1):29-38

51. Verin E, Leroi AM (2009) Poststroke dysphagia rehabilitation by repetitive transcranial magnetic stimulation: a noncontrolled pilot study. Dysphagia 24(2):204-210

52. Du J et al (2016) Repetitive transcranial magnetic stimulation for rehabilitation of poststroke dysphagia: a randomized, double-blind clinical trial. Clin Neurophysiol 127(3):1907-1913

53. Kim L et al (2011) Effect of repetitive transcranial magnetic stimulation on patients with brain injury and dysphagia. Ann Rehabil Med 35(6):765-771

54. Lim KB et al (2014) Effect of low-frequency rTMS and NMES on subacute unilateral hemispheric stroke with dysphagia. Ann Rehabil Med 38(5):592-602

55. Khedr EM, Abo-Elfetoh N, Rothwell JC (2009) Treatment of poststroke dysphagia with repetitive transcranial magnetic stimulation. Acta Neurol Scand 119(3):155-161

56. Michou E et al (2014) Characterizing the mechanisms of central and peripheral forms of neurostimulation in chronic dysphagic stroke patients. Brain Stimul 7(1):66-73

57. Park JW et al (2013) The effect of $5 \mathrm{~Hz}$ high-frequency rTMS over contralesional pharyngeal motor cortex in post-stroke oropharyngeal dysphagia: a randomized controlled study. Neurogastroenterol Motil 25(4):324-e250

58. Khedr EM, Abo-Elfetoh N (2010) Therapeutic role of rTMS on recovery of dysphagia in patients with lateral medullary syndrome and brainstem infarction. J Neurol Neurosurg Psychiatry 81(5):495-499

59. Park E et al (2017) Effects of bilateral repetitive transcranial magnetic stimulation on post-stroke dysphagia. Brain Stimul 10(1):75-82

60. Zhang $\mathrm{C}$ et al (2019) Repetitive transcranial magnetic stimulation in combination with neuromuscular electrical stimulation for treatment of post-stroke dysphagia. J Int Med Res 47(2):662-672

61. Pingue V et al (2018) Dual transcranial direct current stimulation for poststroke dysphagia: a randomized controlled trial. Neurorehabil Neural Repair 32(6-7):635-644

62. Shigematsu T, Fujishima I, Ohno K (2013) Transcranial direct current stimulation improves swallowing function in stroke patients. Neurorehabil Neural Repair 27(4):363-369

63. Yang EJ et al (2012) Effects of transcranial direct current stimulation (tDCS) on post-stroke dysphagia. Restor Neurol Neurosci 30(4):303-311

64. Kumar S et al (2011) Noninvasive brain stimulation may improve stroke-related dysphagia: a pilot study. Stroke 42(4):1035-1040

65. Suntrup $\mathrm{S}$ et al (2015) Electrical pharyngeal stimulation for dysphagia treatment in tracheotomized stroke patients: a randomized controlled trial. Intensive Care Med 41(9):1629-1637

66. Ahn $\mathrm{YH}$ et al (2017) Effect of bihemispheric anodal transcranial direct current stimulation for dysphagia in chronic stroke patients: a randomized clinical trial. J Rehabil Med 49(1):30-35

67. Wang ZY et al (2020) Transcranial direct current stimulation improves the swallowing function in patients with cricopharyngeal muscle dysfunction following a brainstem stroke. Neurol Sci 41(3):569-574

68. Park JW et al (2019) A pilot study of the effects of high-frequency repetitive transcranial magnetic stimulation on dysphagia in the elderly. Neurogastroenterol Motil 31(5):e13561

69. Cosentino G et al (2020) Anodal transcranial direct current stimulation and intermittent theta-burst stimulation improve deglutition and swallowing reproducibility in elderly patients with dysphagia. Neurogastroenterol Motil 32(5):e13791

70. Khedr EM et al (2019) The effect of high-frequency repetitive transcranial magnetic stimulation on advancing Parkinson's 
disease with dysphagia: double blind randomized clinical trial. Neurorehabil Neural Repair 33(6):442-452

71. Chang MC et al (2021) The effect of deep brain stimulation on swallowing function in Parkinson's disease: a narrative review. Dysphagia 36(5):786-799

72. Restivo DA et al (2019) A pilot study on the efficacy of transcranial direct current stimulation applied to the pharyngeal motor cortex for dysphagia associated with brainstem involvement in multiple sclerosis. Clin Neurophysiol 130(6):1017-1024

73 Hamdy S et al (1999) Cortical activation during human volitional swallowing: an event-related fMRI study. Am J Physiol-Gastrointest Liver Physiol 277(1):G219-G225

74. Mosier K et al (1999) Cortical representation of swallowing in normal adults: functional implications. Laryngoscope 109(9):1417-1423

75. Suzuki M et al (2003) Activation of cerebellum and basal ganglia on volitional swallowing detected by functional magnetic resonance imaging. Dysphagia 18(2):71-77

76. Jayasekeran V, Rothwell J, Hamdy S (2011) Non-invasive magnetic stimulation of the human cerebellum facilitates cortico-bulbar projections in the swallowing motor system. Neurogastroenterol Motil 23(9):831-e341

77. Vasant DH et al (2015) High-frequency focal repetitive cerebellar stimulation induces prolonged increases in human pharyngeal motor cortex excitability. J Physiol 593(22):4963-4977

78. Sasegbon A et al (2019) Cerebellar repetitive transcranial magnetic stimulation restores pharyngeal brain activity and swallowing behaviour after disruption by a cortical virtual lesion. J Physiol 597(9):2533-2546

79. Sasegbon A et al (2020) The effects of unilateral and bilateral cerebellar rTMS on human pharyngeal motor cortical activity and swallowing behavior. Exp Brain Res 238(7-8):1719-1733

80. Sasegbon A et al (2021) The effects of midline cerebellar rTMS on human pharyngeal cortical activity in the intact swallowing motor system. Cerebellum 20(1):101-115

81. Erfmann KLC et al (2020) Effects of cerebellar transcranial direct current stimulation (tDCS) on motor skill learning in swallowing. Disabil Rehabil. https://doi.org/10.1080/09638288.2020.1827303

82. Vasant DH et al (2019) Rapid improvement in brain and swallowing behavior induced by cerebellar repetitive transcranial magnetic stimulation in poststroke dysphagia: a single patient case-controlled study. Neurogastroenterol Motil 31(7):e13609
83. Zhong L et al (2021) Repetitive transcranial magnetic stimulation at different sites for dysphagia after stroke: a randomized, observer-blind clinical trial. Front Neurol 12:860

84. Raginis-Zborowska A et al (2019) Genetic influences on the variability of response to repetitive transcranial magnetic stimulation in human pharyngeal motor cortex. Neurogastroenterol Motil 31(7):e13612

85. Michou E et al (2013) Priming pharyngeal motor cortex by repeated paired associative stimulation: implications for dysphagia neurorehabilitation. Neurorehabil Neural Repair 27(4):355-362

86. Cheng I et al (2020) Preconditioning human pharyngeal motor cortex enhances directional metaplasticity induced by repetitive transcranial magnetic stimulation. J Physiol 598(22):5213-5230

87. Ziemann U, Ilić TV, Jung P (2006) Long-term potentiation (LTP)like plasticity and learning in human motor cortex-investigations with transcranial magnetic stimulation (TMS). Supplements to Clinical neurophysiology. Elsevier, pp 19-25

88. Chung CL, Mak MK, Hallett M (2020) Transcranial magnetic stimulation promotes gait training in Parkinson disease. Ann Neurol 88(5):933-945

89. Zimmerman E et al (2020) Motor learning, neuroplasticity, and strength and skill training: moving from compensation to retraining in behavioral management of dysphagia. Am J Speech Lang Pathol 29(2S): 1065-1077

90. Langmore SE, Pisegna JM (2015) Efficacy of exercises to rehabilitate dysphagia: a critique of the literature. Int J Speech Lang Pathol 17(3):222-229

91. Malandraki GA, Johnson S, Robbins J (2011) Functional MRI of swallowing: from neurophysiology to neuroplasticity. Head Neck 33(Suppl 1):S14-20

92 Marchina S et al (2020) Transcranial direct current stimulation for post-stroke dysphagia: a systematic review and meta-analysis of randomized controlled trials. J Neurol 268(1):293-304

93. Unluer NO et al (2019) Effects of low-frequency repetitive transcranial magnetic stimulation on swallowing function and quality of life of post-stroke patients. Dysphagia 34(3):360-371

Publisher's note Springer Nature remains neutral with regard to jurisdictional claims in published maps and institutional affiliations. 